\title{
Evaluation of ALDH1 expression in ipsilateral breast cancer recurrence
}

\author{
TADAHIKO SHIEN $^{1}$, TAKEHIRO TANAKA ${ }^{2}$, MASAHIKO TANABE $^{3}$, YASUHIRO OKUMURA $^{4,5}$, \\ NORIKAZU MASUDA ${ }^{6}$, ATSUSHI YOSHIDA ${ }^{7}$, NOBUYUKI ARIMA ${ }^{5,8}$, \\ YOSHIFUMI KOMOIKE ${ }^{9}$, SATORU TANAKA $^{10}$, TAKUJI IWASE ${ }^{3}$, TETSUYA TAGUCHI $^{11}$, \\ KATSUHIKO NAKATSUKASA ${ }^{11}$, HIDEO INAJI ${ }^{9}$, MAKOTO ISHITOBI ${ }^{9}$ and THE COLLABORATIVE \\ STUDY GROUP OF SCIENTIFIC RESEARCH OF THE JAPANESE BREAST CANCER SOCIETY
}

\begin{abstract}
Departments of ${ }^{1}$ Breast and Endocrine Surgery, and ${ }^{2}$ Pathology, Okayama University Hospital, Okayama 700-8558;
${ }^{3}$ Division of Breast Oncology, The Cancer Institute Hospital of The Japanese Foundation for Cancer Research, Tokyo 135-8550; ${ }^{4}$ Department of Breast and Endocrine Surgery, Kumamoto City Hospital, Kumamoto 862-8505; ${ }^{5}$ Department of Breast Oncology, Department of Pathology, Kumamoto Shinto General Hospital, Kumamoto 862-8655; ${ }^{6}$ Department of Surgery, Breast Oncology, National Hospital Organization Osaka National Hospital, Osaka 540-0006;

${ }^{7}$ Department of Breast Surgery, St. Luke's International Hospital, Tokyo 104-8560; ${ }^{8}$ Department of Pathology, Kumamoto City Hospital, Kumamoto 862-8505; ${ }^{9}$ Department of Breast and Endocrine Surgery, Osaka Medical Center for Cancer and Cardiovascular Diseases, Osaka 537-8511; ${ }^{10}$ Section of Breast and Endocrine Surgery, Department of General and Gastroenterological Surgery, Osaka Medical College, Osaka 569-8686;

${ }^{11}$ Department of Endocrine and Breast Surgery, Kyoto Prefectural University of Medicine, Kyoto 602-0841, Japan
\end{abstract}

Received August 13, 2015; Accepted October 11, 2016

DOI: $10.3892 / 01.2016 .5538$

\begin{abstract}
Aldehyde dehydrogenase 1 (ALDH1) is a cancer stem cell (CSC) marker that is easily evaluable. The expression and clinical significance of ALDH1 in ipsilateral breast tumor recurrence (IBTR) has yet to be investigated. In the present study, the expression profile of ALDH1 and its correlation with prognosis in IBTR tissues was examined. Patients with IBTR from eight institutions were retrospectively enrolled in the study. Immunohistochemistry was used to examine ALDH1 expression patterns in the tissue specimens of primary cancers and IBTRs. ALDH1 expression levels were investigated in 182 IBTR tumors, which included cases of invasive carcinoma selected from 271 consecutive patients with IBTR. ALDH1 was expressed in $23 \%$ of the IBTR tissue samples. The rate of concordant expression between primary cancer and IBTR tissues was $68 \%$. There was no significant association between disease-free survival (DFS) and ALDH1 expression levels in IBTR. IBTRs that expressed ALDH1 and Ki-67 had a poorer prognosis and this expression pattern was significantly
\end{abstract}

Correspondence to: Dr Tadahiko Shien, Department of Breast and Endocrine Surgery, Okayama University Hospital, 2-5-1 Shikata-cho, Kita-ku, Okayama 700-8558, Japan

E-mail: tshien@md.okayama-u.ac.jp

Key words: breast cancer, ipsilateral breast tumor recurrence, aldehyde dehydrogenase 1 , prognostic factor, cancer stem cell associated with DFS ( $\mathrm{P}=0.0073)$. The percentages of ALDH1 positive expression in each tissue subtype were as follows: Luminal A, 20\%; luminal B, 24\%; human epidermal growth factor 2 (HER2), 35\%; triple-negative, 21\%. There was a significant correlation between DFS and ALDH1 expression levels in HER2-type IBTR tissue specimens $(\mathrm{P}=0.034)$. In conclusion, it is possible that ALDH1 and $\mathrm{Ki}-67$ expression levels may be useful for predicting prognosis in patients with HER2-type tumors.

\section{Introduction}

Breast conserving surgery (BCS) is a standard procedure for the treatment of early-stage breast cancer (1). It has been demonstrated in previous studies that the 10-year overall survival (OS) rate of patients treated with BCS (65\%) is similar to that of those treated with mastectomy $(66 \%)(1,2)$; however, there is a higher risk of ipsilateral breast tumor recurrence (IBTR) following BCS (3). As IBTR is associated with an increased risk of distant disease and mortality, adjuvant radiation therapy of the residual breast tissue following BCS is often required to decrease the risk of IBTR (3). Furthermore, adjuvant hormone therapy and chemotherapy, which are used for the majority of patients, may prevent IBTR (3); however, a considerable number of IBTR cases are diagnosed following BCS administered with an adjuvant therapy $(4,5)$.

The risk factors for developing IBTR have yet to be determined, and further studies of the tumor biology of IBTR are required to identify tumor characteristics that may be used in the selection of an appropriate treatment strategy. A previous 
study revealed a significant difference in distant disease-free survival following IBTR depending on the subtype of breast cancer tissues present, as determined by immunohistochemical staining (6). In particular, increased or persistently high $\mathrm{Ki}-67$ expression levels in cases of IBTR were significantly associated with a poorer prognosis (7).

A predictive prognostic factor that has gained significant interest in association with breast cancer is the presence of cancer stem cells (CSCs). CSCs have self-renewal and multi-lineage differentiation capacities and are frequently resistant to conventional anti-cancer drug and radiation therapies (8). The presence of CSCs may be an important factor in cases of IBTR occurring subsequent to BCS and adjuvant therapy. However, to the best of our knowledge, there are no previous studies that have discussed the presence of CSCs within IBTR tissues. Aldehyde dehydrogenase 1 (ALDH1) is a marker of CSCs (9) that may be easily evaluated in primary breast cancer tissues and is a potentially useful prognostic factor. In the current study, the expression levels of ALDH1 in the primary lesion and in IBTR tissues were investigated, and their association with other clinicopathological factors and the prognostic impact of ALDH1 in IBTR was determined.

\section{Materials and methods}

Patients and samples. A total of 271 consecutive patients with histologically diagnosed IBTR without distant metastases, who underwent definitive surgery for IBTR between 1989 and 2008, were recruited from eight institutions (Okayama University Hospital, Okayama; The Cancer Institute Hospital of the Japanese Foundation for Cancer Research, Tokyo; Kumamoto City Hospital, Kumamoto National Hospital Organization; Osaka National Hospital, Osaka; St. Luke's International Hospital, Tokyo; Osaka Medical Center for Cancer and Cardiovascular Diseases, Osaka; Osaka Medical College, Osaka; Kyoto Prefectural University of Medicine, Kyoto) in Japan. Each institution's review board approved this retrospective study. The inclusion criteria for were as follows: i) The patient was undergoing BCS and axillary surgery (sentinel lymph node biopsy was only permitted if the nodes had no metastasis); ii) IBTR was histologically determined; and iii) the patient was undergoing definitive surgery for IBTR at a time prior to the year 2008. The exclusion criteria were as follows: i) Presence of synchronous metastases (defined as occurring within three months); ii) bilateral breast cancer; iii) history of prior malignancies other than breast cancer; and iv) presence of tumors located in the skin or muscle only, without associated parenchymal disease. Of the 271 IBTR cases, 182 met the criteria and were included in the present study, which investigated the frequency and prognostic impact of the ALDH1 expression profile in IBTR and primary breast cancer tissue.

Immunohistological examination. Estrogen receptor (ER) and progesterone receptor $(\mathrm{PgR})$ status were determined by immunohistochemistry (IHC) (7), and those tumors with $\geq 10 \%$ positively stained tumor cells were classified as being positive for ER. Tissue samples were considered positive for human epidermal growth factor receptor 2 (HER2) if scored as $3+$, or if fluorescence in situ hybridization identified a
HER2/chromosome 17 ratio of $>2.0$ (7). The ER and HER2 status in each tissue sample were evaluated independently in each institution. Proliferation activity was assessed by immunostaining with the Ki-67 antibody (clone MIB-1, cat. no., M7240; dilution, 1:100; Dako, Glostrup, Denmark) using an autostainer (Benchmark XT; Ventana Medical Systems, Inc., Tucson, AZ, USA). Ki-67 staining was centrally evaluated by one pathologist (Kumamoto City Hospital) who was blinded to the clinical data. The proportion of proliferating cells was determined by counting $\geq 500$ tumor cells in hot spots, defined as having a dense concentration of positive cancer nuclei in each tumor according to a previously described protocol $(10,11)$ Breast cancer tissues were classified using the IHC results, according to a previously described protocol (6), into the following subtypes: Triple-negative (ER-, PgR- and HER2-negative); HER2 (HER2-positive, ER- and PgR-negative); luminal A (ER- and/or PgR-positive, HER2-negative and Ki-67 <15\%); and luminal B (ER-positive, HER2-negative and Ki-67 $\geq 15 \%$, or ER-positive and HER2-positive).

IHC was used to evaluate the ALDH1 expression levels in the surgical tissue specimens of primary cancer and IBTR cases that were identified to be invasive carcinomas. The antibody used was anti-ALDH1 (clone 44; \#611195; dilution, 1:250; BD Biosciences, Franklin Lakes, NJ, USA). Imaging analysis of ALDH1 expression in the breast tumor tissues was performed using fluorescence microscopy with one selected high-power field (magnification, 400x; Olympus BX53; Olympus Corporation, Tokyo, Japan) per case, as the immunoreactivity of ALDH-1 was approximately homogeneous with the results from a previous study (12-14). The percentage of ALDH1-positive cells was determined as described in previous studies (12-14), in which the tumor specimens were classified into positive and negative groups based on the IHC staining for ALDH1, with positive defined as $>1 \%$ of tumor cells exhibiting positive staining. Representative results of IHC staining for ALDH1 in breast cancer tissues are presented in Fig. 1.

Statistical analysis. Disease-free survival (DFS) was calculated as the duration from the time of initial surgery for IBTR to the diagnosis of a recurrence. Differences in clinicopathological data were compared using the $\chi^{2}$ test. For comparison between patients with recurrent disease and the recurrence-free patients, the odds ratios for differing variables were assessed by applying a logistic regression model for univariate and multivariate analyses. Survival curves were calculated using the Kaplan-Meier method, and the log-rank test was used to evaluate the statistical significance of the differences in survival among the patient subgroups. Varations between overall survival curves were determined using a log-rank test. For univariate and multivariate analyses, the Cox regression method was used to evaluate the influence of the variables on survival. All of the data were analyzed with the use of JMP version 11.0.0 statistical software (SAS Institute, Cary, NC, USA). $\mathrm{P}<0.05$ was considered to indicate a statistically significant difference.

\section{Results}

Patient characteristics. Patient clinical characteristics are presented in Table I. A total of 271 patients were registered 
Table I. Clinical characteristics of the patients.

A, Patient and tumor characteristics

\begin{tabular}{|c|c|c|}
\hline \multirow[b]{2}{*}{ Characteristic } & \multicolumn{2}{|c|}{ Value } \\
\hline & Primary tumor & IBTR \\
\hline Age, years; median (range) & $46(26-84)$ & $51(29-88)$ \\
\hline $\begin{array}{l}\text { Median primary tumor } \\
\text { size, } \mathrm{cm} \text { (range) }\end{array}$ & $2(0-6.8)$ & - \\
\hline \multicolumn{3}{|l|}{$\mathrm{ER}, \mathrm{n}(\%)$} \\
\hline Positive & $101(55)$ & $113(62)$ \\
\hline Negative & $64(35)$ & $69(38)$ \\
\hline Unknown & $17(9)$ & - \\
\hline \multicolumn{3}{|l|}{ PgR, n (\%) } \\
\hline Positive & $83(46)$ & $78(43)$ \\
\hline Negative & $69(38)$ & $104(57)$ \\
\hline Unknown & $30(16)$ & - \\
\hline \multicolumn{3}{|l|}{ HER2, n (\%) } \\
\hline Positive & $20(11)$ & $40(22)$ \\
\hline Negative & $96(53)$ & $142(78)$ \\
\hline Unknown & $66(36)$ & - \\
\hline \multicolumn{3}{|l|}{ Ki-67, n (\%) } \\
\hline$\geq 15 \%$ & - & $109(60)$ \\
\hline$<15 \%$ & - & $73(40)$ \\
\hline
\end{tabular}

$\mathrm{B}$, Treatment and survival characteristics

\begin{tabular}{lc}
\hline Characteristic & Value \\
\hline Adjuvant treatment, n (\%) & \\
Chemotherapy & $61(34)$ \\
Hormone therapy & $105(58)$ \\
Trastuzumab & $5(3)$ \\
Adjuvant radiation therapy & $90(51)$ \\
Disease-free survival time, & $46(2-206)$ \\
months; median (range) &
\end{tabular}

ER, estrogen receptor; PgR, progesterone receptor; HER2, human epidermal growth factor receptor 2; IBTR, ipsilateral breast tumor recurrence.

for the current study, of which 182 IBTR tissue specimens were examined for the expression of ALDH1. The median ages of the patients at the time of initial breast surgery and surgery for IBTR were 46 years (range, 26-84 years) and 51 years (range, 29-88 years), respectively. In the primary tumor tissues, the proportions of cells that were positive for ER, PgR and HER2 were 55, 46 and 11\%, respectively; these values were 62,43 and $22 \%$ in the IBTR tissue samples. Following primary surgery, $10 \%$ of patients exhibited a positive surgical margin. Adjuvant radiation therapy was administered to $51 \%$ of the patients. The median duration from initial surgery to a diagnosis of IBTR was 46 months (range, 2-206 months).
Table II. Expression profile of ALDH1 in breast cancer.

\begin{tabular}{lcc}
\hline ALDH1 expression & Primary tumor, n (\%) & IBTR, n (\%) \\
\hline 0 & $114(63)$ & $139(76)$ \\
$1+$ & $29(16)$ & $30(16)$ \\
$2+$ & $4(2)$ & $7(4)$ \\
$3+$ & $4(2)$ & $6(3)$ \\
Unknown & $31(17)$ & $0(0)$ \\
\hline
\end{tabular}

ALDH1, aldehyde dehydrogenase 1; IBTR, ipsilateral breast tumor recurrence; $1+, 1-5 \%$ positively stained cells; $2+, 6-10 \%$ positively stained cells; $3+,>10 \%$ positively stained cells.

Table III. Concordance of ALDH1 expression in primary tumor and IBTR tissues.

\begin{tabular}{llr}
\hline & \multicolumn{1}{c}{$\begin{array}{c}\text { ALDH1 expression in } \\
\text { primary tumor, } \mathrm{n}(\%)\end{array}$} \\
\cline { 2 - 3 } $\begin{array}{l}\text { ALDH1 expression } \\
\text { in IBTR }\end{array}$ & Negative & Positive \\
\hline Negative & $92(61)$ & $22(15)$ \\
Positive & $26(17)$ & $11(7)$ \\
\hline
\end{tabular}

ALDH1, aldehyde dehydrogenase 1; IBTR, ipsilateral breast tumor recurrence.

ALDH1 expression in primary tumor and IBTR. The expression levels of ALDH1 in the primary tumor and IBTR tissues are presented in Table II. A total of 37 (20\%) primary tumor tissue samples and $43(23 \%)$ IBTR tissue specimens were ALDH1 positive ( $>1 \%$ positively stained cells). As a minority of tissue samples were classified as $2+$ (primary tumor, $2 \%$; IBTR, $4 \%$ ) or $3+$ (primary tumor, $2 \%$; IBTR, 3\%), it was determined that tissues with $>1 \%$ ALDH1-positive cells would be considered positive cases. The concordance of the ALDH1 expression status between the primary tumor and IBTR cases is presented in Table III; the total concordance rate was $68 \%$.

Correlation between ALDH1/Ki-67 expression and disease-free survival. There was no significant association observed between the rate of DFS following IBTR and ALDH1 expression status in IBTR tissues (Fig. 2); however, patients who exhibited ALDH1-positive tissues in the primary tumor and the IBTR had the poorest DFS (Fig. 3). Multivariate analysis revealed that $\mathrm{Ki}-67 \geq 15 \%$ (odds ratio, 2.31; $\mathrm{P}=0.003$ ) and ER-positive status (odds ratio, $0.48 ; \mathrm{P}=0.027$ ) were statistically significant predictive factors for DFS following IBTR (Table IV); however, ALDH1 did not have prognostic value $(\mathrm{P}=0.513)$.

According to ALDH1 and Ki-67 statuses, the tissue samples were further subdivided (Fig. 4). The ALDH1-negative patients with low Ki-67 expression $(<15 \%)$ had typically better outcomes compared with those patients exhibiting high Ki-67 expression levels $(\geq 15 \%)$. However, there were no significant 

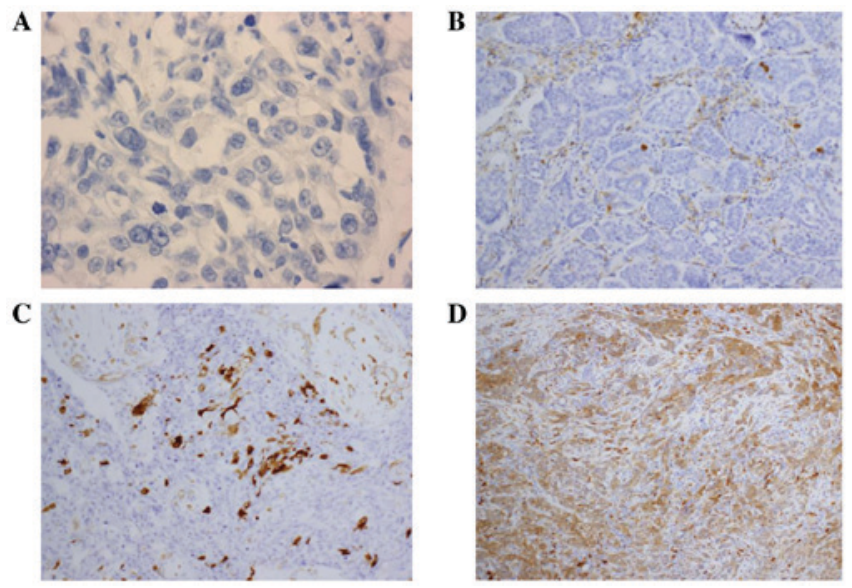

Figure 1. Evaluation of ALDH1 expression. ALDH1 expression was examined by immunohistochemistry in the surgical specimens obtained during the treatment of primary breast cancer and ipsilateral breast tumor recurrence, which were invasive carcinoma tissues. Images show representative ALDH1 immunostaining results in the breast cancer tissues: (A) Negative; (B) $1+(1-5 \%$ positive cells); (C) $2+(5-10 \%$ positive cells $)$; (D) $3+(>10 \%$ positive cells). ALDH1, aldehyde dehydrogenase 1.

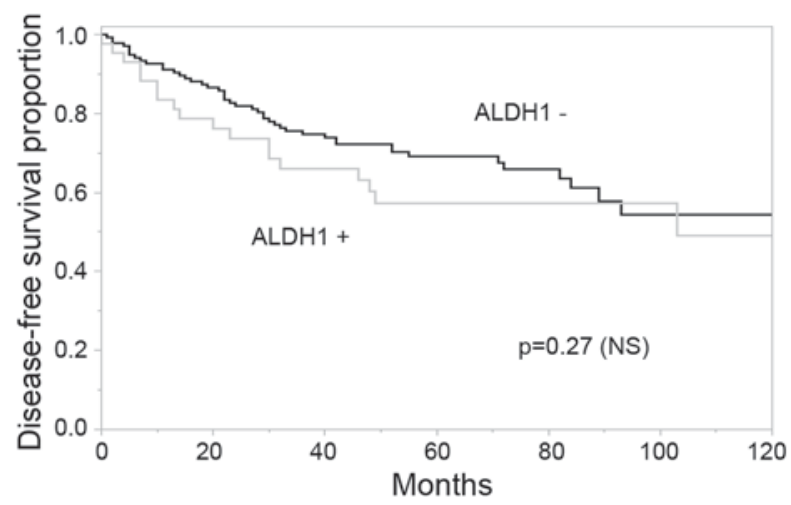

Figure 2. Kaplan-Meier curves revealing the correlation between ALDH1 expression in IBTR tissue and DFS following IBTR. There was no significant association between the DFS following IBTR and ALDH1 expression in IBTR tissues. ALDH1, aldehyde dehydrogenase 1; IBTR, ipsilateral breast tumor recurrence; DFS, disease-free survival.

differences observed between the Ki-67 <15\%, ALDH1-positive group and the Ki-67 <15\%, ALDH1-negative group.

ALDH1 expression according to subtype. When classified according to ER, HER 2 and Ki-67 expression profiles, the subtype distribution of IBTR was as follows: Luminal A, 51 patients (28\%); luminal B, 62 patients (34\%); HER2, 26 patients (14\%); and triple-negative, 43 patients $(24 \%)$. The proportions of each of the subtypes that were positive for ALDH1 were as follows: Luminal A, 20\%; luminal B, 24\%; HER2, 35\%; and triple-negative, 21\% (Table V). The proportions of patients that received adjuvant drug therapy following IBTR for luminal A, luminal B, HER2 and triple-negative types were 93, 92, 58 and $56 \%$, respectively. A total of 7 patients (27\%) with the HER2 subtype received trastuzumab following IBTR surgery.

There was no significant correlation observed between ER, PgR, HER2 or Ki-67 expression status and ALDH1 expression status, or between ALDHI and the specific tissue subtype
Table IV. Multivariate analysis using the Cox proportional hazards model to identify predictors of disease-free survival.

\begin{tabular}{lccc}
\hline Variable & Odds ratio & $95 \%$ CI & P-value* $^{*}$ \\
\hline ALDH1-positive & 1.21 & $0.676-2.065$ & 0.513 \\
Ki-67 $\geq 15 \%$ & 2.31 & $1.320-4.260$ & 0.003 \\
ER-positive & 0.48 & $0.233-0.920$ & 0.027 \\
PgR-positive & 0.93 & $0.457-1.959$ & 0.846 \\
HER2-positive & 0.78 & $0.410-1.430$ & 0.436 \\
\hline
\end{tabular}

CI, confidence interval; ALDH1, aldehyde dehydrogenase 1; ER, estrogen receptor; PgR, progesterone receptor; HER2, human epidermal growth factor receptor 2 .

Table V. Expression of ALDH1 according to ER and HER2 status in cases of ipsilateral breast tumor recurrence.

\begin{tabular}{|c|c|c|c|}
\hline Category & Total cases, $\mathrm{n}$ & $\begin{array}{l}\text { ALDH1-positive } \\
\text { cases, n }(\%)\end{array}$ & P-value* \\
\hline ER & & & 0.54 \\
\hline Positive & 113 & $25(22)$ & \\
\hline Negative & 69 & $18(26)$ & \\
\hline PgR & & & 0.84 \\
\hline Positive & 104 & $24(23)$ & \\
\hline Negative & 78 & $19(24)$ & \\
\hline HER 2 & & & 0.29 \\
\hline Positive & 40 & $12(30)$ & \\
\hline Negative & 142 & $31(22)$ & \\
\hline Ki-67 & & & 0.14 \\
\hline$\geq 15 \%$ & 109 & $30(28)$ & \\
\hline$<15 \%$ & 73 & $13(18)$ & \\
\hline Subtype & & & 0.52 \\
\hline Luminal $\mathrm{A}^{\mathrm{a}}$ & 51 & $10(20)$ & \\
\hline Luminal $\mathrm{B}^{\mathrm{b}}$ & 62 & $15(24)$ & \\
\hline HER2 type ${ }^{c}$ & 26 & $9(35)$ & \\
\hline Triple negative $^{\mathrm{d}}$ & 43 & $9(21)$ & \\
\hline
\end{tabular}

P-values were calculated using univariate analysis. ${ }^{\mathrm{a}} \mathrm{ER}$ and/or PgR-positive, HER2-negative and Ki-67 $\leq 14 \%$; ${ }^{\mathrm{b}} \mathrm{ER}$ and/or PgR-positive, HER2-negative and Ki-67 $\geq 15 \%$, or ER and/or PgR-positive and HER2-positive; 'ER and PgR-negative and HER2-positive; ${ }^{\mathrm{d}} \mathrm{ER}$ and PgR-negative and HER2-negative. ALDH1, aldehyde dehydrogenase 1; ER, estrogen receptor; PgR, progesterone receptor; HER2, human epidermal growth factor receptor 2; IBTR, ipsilateral breast tumor recurrence.

(Table V). Furthermore, there was no significant association between the administration of adjuvant therapy following primary surgery and ALDH1 expression levels in IBTR tissues (Table VI).

When the prognosis was analyzed with regard to ALDH1 expression and tissue subtype (Fig. 5A-D), the expression status of ALDH1 in IBTR tissues of a HER2 subtype was observed to be a significant prognostic factor for DFS ( $\mathrm{P}=0.034$; Fig. 5C). 
Table VI. Association between adjuvant therapy administered following the primary surgery and ALDH1 expression levels in IBTR tissues.

\begin{tabular}{lcc}
\hline Category & ALDH1-positive cases, n (\%) & P-value \\
\hline RT & $19(21)$ & 0.21 \\
+ & $24(27)$ & \\
- & & 0.73 \\
CT and/or HT & $34(25)$ & \\
+ & $7(20)$ & \\
- & & \\
\hline
\end{tabular}

P-values were calculated using the $\chi^{2}$ test. ALDH1, aldehyde dehydrogenase 1; IBTR, ipsilateral breast tumor recurrence; RT, adjuvant radiation therapy; $\mathrm{CT}$, adjuvant chemotherapy including molecular targeted therapy; HT, adjuvant hormone therapy.

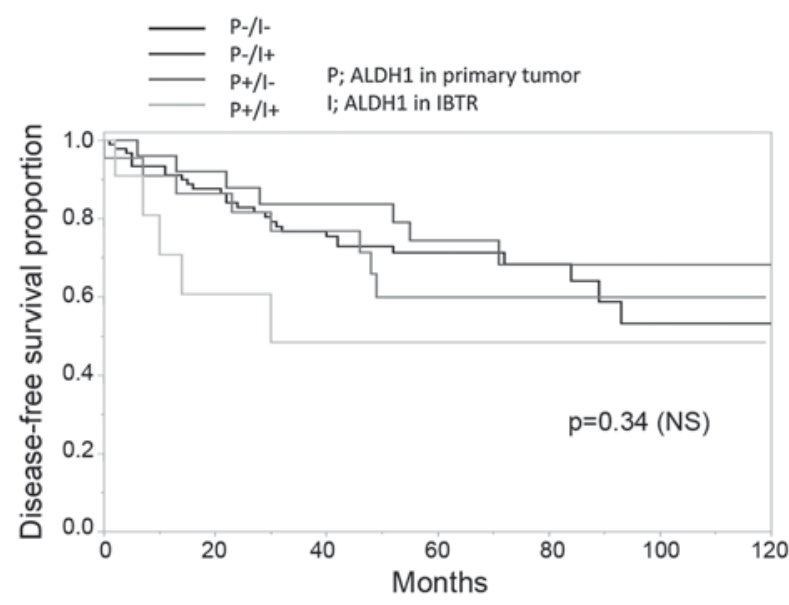

Figure 3. Kaplan-Meier curves presenting the correlation between ALDH1 expression and DFS following IBTR. Patients with ALDH1-positive tissues both in the primary tumor and the IBTR had the poorest DFS. ALDH1, aldehyde dehydrogenase 1; IBTR, ipsilateral breast tumor recurrence; PT, ALDH1 expression in primary tumor; I, ALDH1 expression in IBTR; DFS, disease-free survival.

There was no significant difference observed in the rates of adjuvant trastuzumab administration between the ALDH1-positive and -negative groups (ALDH1-positive, 25\%; ALDH1-negative, 16\%).

\section{Discussion}

To the best of our knowledge, the present study is the first to investigate the frequency and prognostic value of ALDH1 expression in IBTR tissues. The cut-off level for the classification of positive ALDH1 expression was set at $>1 \%$ in the current study, as there is no standardized cut-off level or method currently available. The ALDH1 positive cells were counted in hot spots, and positive ALDH1 expression was detected in $20 \%$ of the primary tumor samples and $23 \%$ of the IBTR tissue samples. The frequency of patients with ALDH1-expressing primary breast cancer (20\%) in the current study was similar to that observed in previous studies

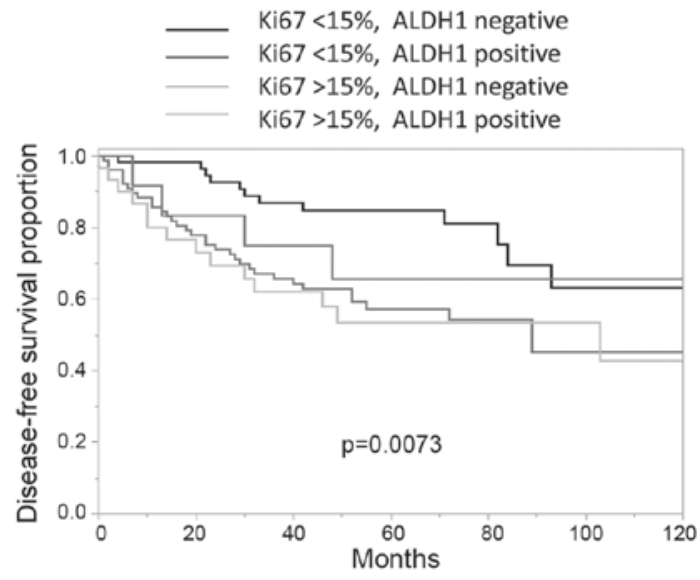

Figure 4. Kaplan-Meier curves revealing the correlation between ALDH1/Ki-67 expression in IBTR and disease-free survival. The patients with low Ki-67 expression $(<15 \%)$ and ALDH1-negative patients had improved outcomes, compared with those patients exhibiting high Ki-67 expression levels (>15\%). ALDH1, aldehyde dehydrogenase 1. IBTR, ipsilateral breast tumor recurrence.

$(19,17.5 \%)(10,11)$; however, the ALDH1 expression the in IBTR group (23\%) was higher. It was hypothesized that this variation was due to the dissimilarities between the IBTR and primary tumors. IBTRs included new primary tumors and true recurrences following adjuvant therapy. Therefore, ALDH1-positive cancer stem cells within true recurrence were able to survive following adjuvant systemic and radiation therapy and may be not similar to the cancer cells within the primary tumor. A previous study revealed that the frequency of ALDH1 positive cells in metastatic tumors is relatively high compared with primary tumors (14). This discrepancy may be due to there being no definitive ALDH1 cut-off level; the cut-off level $(>1 \%)$ used for the present study was lower than the value used in previous reports (12-14). The total number of IBTR tissue samples used in the current study was relatively low, compared with previous reports that analyzed ALDH1 expression in primary breast tumors. Therefore, the associations between ALDH1 expression, tumor subtypes and clinical data could not be analyzed.

A similar expression rate for ALDH1 was observed in the primary tumor and IBTR tissue specimens. The concordance rate between ALDH1 expression in primary tumors and associated IBTR was $68 \%$. It was previously reported that variation in the ER, HER2 and Ki-67 statuses between the primary tumors and IBTR tissues was observed in 16.2, 13.7 and $36.8 \%$ of cases, respectively (7). The discordance rates for ALDH1 expression and Ki-67 status were higher, compared with ER, PgR and HER2 in our previous study (14). A number of previous studies have evaluated the variations between primary tumors and distant metastases and presented similar results to those observed in the current study $(12,15,16)$. The discordance rates for the expression of ER, HER2, Ki-67 and ALDH1 between primary breast tumor tissues and lung metastases were 0,21, 43 and 50\%, respectively (14), and the corresponding rates for primary breast tumor tissue and axillary lymph node metastases were 12.5, 17.5, 22.5 and $42.5 \%$ (12), respectively. It was hypothesized that a reason for the variation in the expression of these specific biomarkers 

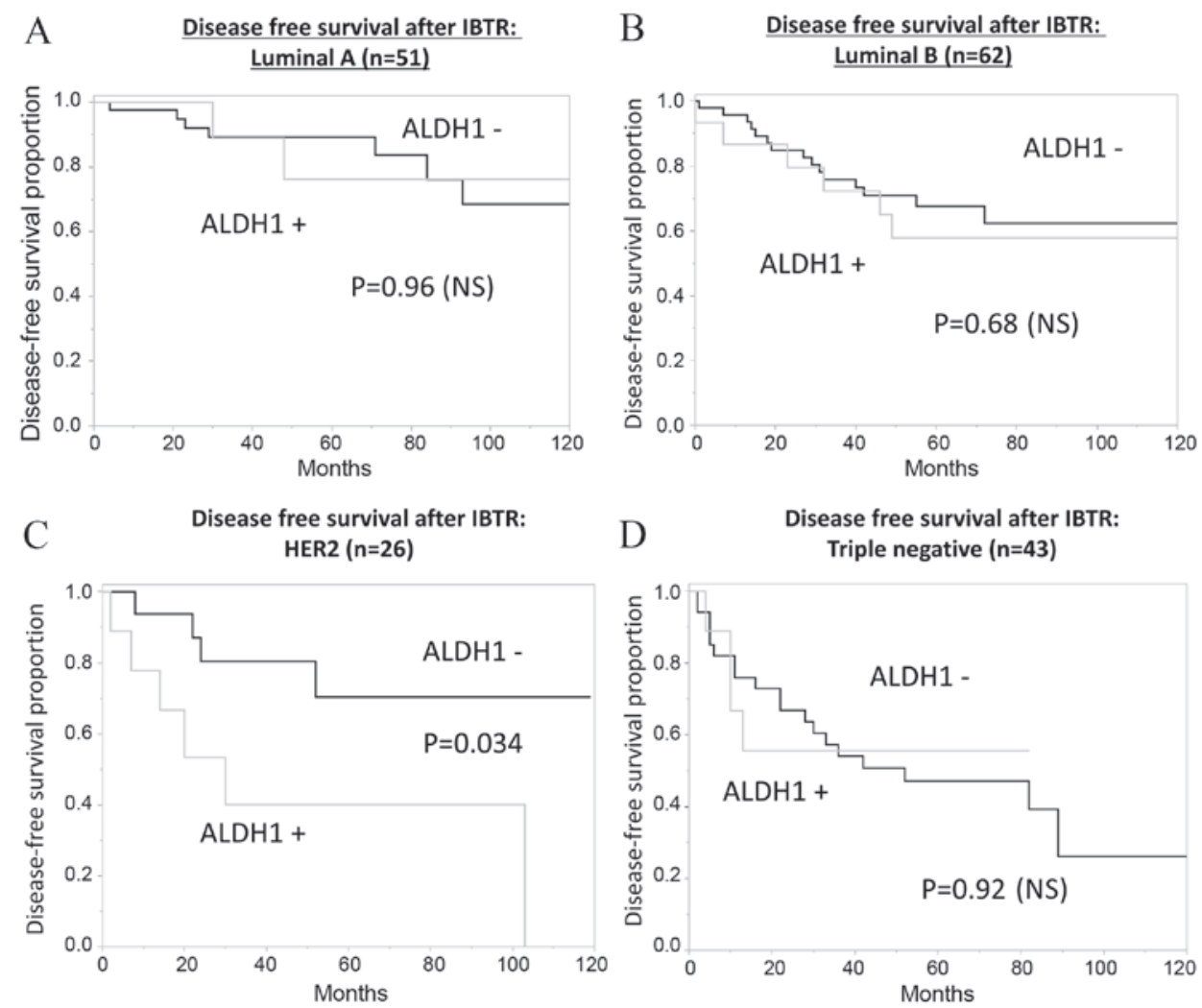

Figure 5. Kaplan-Meier curves presenting the correlation between ALDH1 expression in IBTR and DFS according to subtype of IBTR: (A) Luminal A (n=51); (B) luminal B ( $\mathrm{n}=62)$; (C) HER2 ( $\mathrm{n}=26)$; (D) triple negative ( $\mathrm{n}=43$ ). ALDH1 expression in IBTR tissues of HER2 subtype was a significant prognostic factor for DFS ( $\mathrm{P}=0.034)$. ALDH1, aldehyde dehydrogenase 1; IBTR, ipsilateral breast tumor recurrence; DFS, disease-free survival; HER2, human epidermal growth factor receptor 2.

may be the exposure of metastatic tumor tissues to adjuvant systemic chemotherapy or radiation therapy (17).

Tanei et al (18) identified a difference in ALDH1 expression levels in breast tumor tissues prior to and following neoadjuvant chemotherapy (NAC). Although the association between adjuvant therapy and ALDH1 expression levels in IBTR tissues was investigated in the current study, no significant correlation was demonstrated. However, as the current study was retrospective, further evaluation may be required. The present study also included a mixture of patient tissue samples consisting of 'true IBTR' and 'new primary tumors'; new primary tumors are not expected to have any correlation with the first primary tumor (19-21). The timing of diagnosis of IBTR was distinct from that of the diagnosis following NAC. The post-NAC surgery was performed immediately following the end of systemic therapy. However, the duration between the administration of systemic therapies and time of surgery for IBTR were not homogeneous. There were many cancer stem cells present in tissue specimens following NAC administration, as they are resistant to systemic therapy (18). Therefore, ALDH1 expression levels in IBTR may be varied according to the timing of surgery following systemic therapy.

In previous reports, ALDH1 expression in primary breast tumors was identified to be an independent predictor of early tumor relapse of invasive ductal carcinoma (22), particularly in patients with triple-negative breast cancer $(23,24)$. In the present study, no significant correlation was observed between ALDH1 expression in primary tumors or IBTR tissues, and the prognosis of patients with IBTR. However, the results suggested that low Ki-67 and high ER expression levels in IBTR tissues were significantly associated with improved prognosis. Furthermore, the expression levels of $\mathrm{Ki}-67$ and ALDH1 in IBTR tissues were determined to be significant predictors of poor prognosis and a short DFS time.

Subgroup analysis identified no significant correlation between the tumor subtype and ALDH1 expression levels; however, the expression levels of ALDH1 were significantly associated with prognosis in HER2-positive IBTR, possibly due to HER2-positive IBTR tissue including numerous new primary tumors. In the current study, the rate of HER2 expression in IBTRs (22\%) was higher than that in primary tumors (11\%), and 54\% of primary tumors in HER2-type IBTR tissues were diagnosed as HER2-negative or unknown. Adjuvant trastuzumab therapy was administered to three patients. Adjuvant trastuzumab following primary surgery is recommended for patients with HER2-type breast cancer (25); however, no prospective studies have been conducted to examine the efficacy of adjuvant trastuzumab for IBTR. A total of $74 \%$ of the patients with HER2-type IBTR did not receive trastuzumab. A number of patients with luminal A or B subtypes of breast cancer received adjuvant hormonal therapy. Our previous study reported that the IBTR tumor subtype is more important in predicting treatment outcomes than the subtype of the primary tumor (6). There was no significant difference in the proportions of patients that received adjuvant trastuzumab treatment between cases of ALDH1-positive and ALDH1-negative IBTR 
in the present study. Therefore, trastuzumab may reduce the variation in disease free survival between patients with ALDH1-positive and ALDH1-negative IBTR.

In the present study, only tissue specimens from patients with IBTR were evaluated and no control specimens from breast cancer without IBTR were included. Therefore, a comparison between patients with and without IBTR is required in future studies. Further studies are required to evaluate the value of ALDH1 expression as a predictor for IBTR. It is possible that ALDH1 expression levels in primary tumors are not a significant predictor of IBTR, as it was identified that the expression levels of ALDH1 in the primary tumor tissue samples of the patients with IBTR were not higher compared with previous studies of patients with primary breast tumors. Further studies are required to investigate this.

In conclusion, the expression profile of ALDH1 in IBTR tissue specimens was retrospectively analyzed and ALDH1 was not demonstrated to be an independent prognostic factor. However, ALDH1 expression levels may be a useful prognostic factor in certain subgroups or when examined in combination with other factors, including Ki67 or breast cancer subtypes. Further studies regarding the prognostic importance of ALDH1 expression levels in IBTR are required, with a larger sample number and a prospective approach.

\section{Acknowledgements}

This study was supported in part by Grants-in-Aid for Scientific Research from the Japanese Breast Cancer Society (grant no. H25-26), and the Health and Labour Sciences Research Expenses for Commission, Applied Research for Innovative Treatment of Cancer (grant no. H26-applied-general-043) from the Ministry of Health, Labour and Welfare, and Japan Agency for Medical Research and Development.

\section{References}

1. van Dongen JA, Voogd AC, Fentiman IS, Legrand C, Sylvester RJ, Tong D, van der Schueren E, Helle PA, van Zijl K and Bartelink H: Long-term results of a randomized trial comparing breast-conserving therapy with mastectomy: European organization for research and treatment of cancer 10801 trial. J Natl Cancer Inst 92: 1143-1150, 2000.

2. Morris AD, Morris RD, Wilson JF, White J, Steinberg S, Okunieff P, Arriagada R, Lê MG, Blichert-Toft $M$ and van Dongen JA: Breast-conserving therapy vs mastectomy in early-stage breast cancer: A meta-analysis of 10-year survival. Cancer J Sci Am 3: 6-12, 1997.

3. Clarke M, Collins R, Darby S, Davies C, Elphinstone P, Evans V, Godwin J, Gray R, Hicks C, James S, et al: Effects of radiotherapy and of differences in the extent of surgery for early breast cancer on local recurrence and 15-year survival: An overview of the randomised trials. Lancet 366: 2087-2106, 2005.

4. Veronesi U, Cascinelli N, Mariani L, Greco M, Saccozzi R, Luini A, Aguilar M and Marubini E: Twenty-year follow-up of a randomized study comparing breast-conserving surgery with radical mastectomy for early breast cancer. N Engl J Med 347: 1227-1232, 2002.

5. Fisher B, Anderson S, Bryant J, Margolese RG, Deutsch M, Fisher ER, Jeong JH and Wolmark N: Twenty-year follow-up of a randomized trial comparing total mastectomy, lumpectomy, and lumpectomy plus irradiation for the treatment of invasive breast cancer. N Engl J Med 347: 1233-1241, 2002.

6. Ishitobi M, Okumura Y, Arima N, Yoshida A, Nakatsukasa K, Iwase T, Shien T, Masuda N, Tanaka S, Tanabe M, et al: Breast cancer subtype and distant recurrence after ipsilateral breast tumor recurrence. Ann Surg Oncol 20: 1886-1892, 2013.
7. Okumura Y,Nishimura R, Nakatsukasa K, Yoshida A, Masuda N, Tanabe M, Shien T, Tanaka S, Arima N, Komoike Y, et al: Change in estrogen receptor, HER 2 and Ki-67 status between primary breast cancer and ipsilateral breast cancer tumor recurrence. Eur J Surg Oncol 41: 548-552, 2015.

8. Wicha MS, Liu S and Dontu G: Cancer stem cells: An old idea-a paradigm shift. Cancer Res 66: 1883-1890, 1895-1896, 2006.

9. Ginestier C, Hur MH, Charafe-Jauffret E, Monville F, Dutcher J, Brown M, Jacquemier J, Viens P, Kleer CG, Liu S, et al: ALDH1 is a marker of normal and malignant human mammary stem cells and a predictor of poor clinical outcome. Cell Stem Cell 1: 555-567, 2007.

10. Arima N, Nishimura R, Osako T, Nishiyama Y, Fujisue M, Okumura Y, Nakano M, Tashima R and Toyozumi Y: The importance of tissue handling of surgically removed breast cancer for an accurate assessment of the Ki-67 index. J Clin Pathol 69:255-259, 2016.

11. Arima N, Nishimura R, Osako T, Nishiyama Y, Fujisue M, Okumura Y, Nakano M, Tashima R and Toyozumi Y: A comparison of the hot spot and the average cancer cell counting methods and the optimal cutoff point of the $\mathrm{Ki}-67$ index for luminal type breast cancer. Oncology 90:43-50, 2016.

12. Nogami T, Shien T, Tanaka T, Nishiyama K, Mizoo T, Iwamto T, Ikeda H, Taira N, Doihara H and Miyoshi S: Expression of ALDH1 in axillary lymph node metastases is a prognostic factor of poor clinical outcome in breast cancer patients with 1-3 lymph node metastases. Breast Cancer 21: 58-65, 2014.

13. Ito M, Shien T, Omori M, Mizoo T, Iwamoto T, Nogami T, Motoki T, Taira N, Doihara H and Miyoshi S: Evaluation of aldehyde dehydrogenase 1 and transcription factors in both primary breast cancer and axillary lymph node metastases as a prognostic factor. Breast Cancer 23: 437-444, 2016.

14. Nogami T, Shien T, Tanaka T, Doihara H, Taira N, Takabatake D, Nishimura R, Nishiyama K, Mizoo T and Ohsumi S: The discordance between primary breast cancer lesions and pulmonary metastatic lesions in expression of aldehyde dehydrogenase 1-positive cancer cells. Breast Cancer 21: 698-702, 2014.

15. Yeung C, Hilton J, Clemons M, Mazzarello S, Hutton B, Haggar F, Addison CL, Kuchuk I, Zhu X, Gelmon K and Arnaout A: Estrogen, progesterone, and HER2/neu receptor discordance between primary and metastatic breast tumours-a review. Cancer Metastasis Rev 35: 427-437, 2016.

16. Rossi S, Basso M, Strippoli A, Dadduzio V, Cerchiaro E, Barile R, D'Argento E, Cassano A, Schinzari G and Barone C: Hormone receptor status and HER2 expression in primary breast cancer compared with synchronous Axillary metastases or recurrent metastatic disease. Clin Breast Cancer 15: 307-312, 2015.

17. Shien K, Toyooka S, Ichimura K, Soh J, Furukawa M, Maki Y, Muraoka T, Tanaka N, Ueno T, Asano H, et al: Prognostic impact of cancer stem cell-related markers in non-small cell lung cancer patients treated with induction chemoradiotherapy. Lung Cancer 77: 162-167, 2012.

18. Tanei T, Morimoto K, Shimazu K, Kim SJ, Tanji Y, Taguchi T, Tamaki Y and Noguchi S: Association of breast cancer stem cells identified by aldehyde dehydrogenase 1 expression with resistance to sequential paclitaxel and epirubicin-based chemotherapy for breast cancers. Clin Cancer Res 15: 4234-4241, 2009.

19. Benson JR and della Rovere GQ: Ipsilateral breast cancer recurrence. Breast 17: 12-18, 2008

20. Chen SL and Martinez SR: The survival impact of the choice of surgical procedure after ipsilateral breast cancer recurrence. Am J Surg 196: 495-499, 2008.

21. McGrath S, Antonucci J, Goldstein N, Wallace M, Mitchell C, Grills I, Jolly S, Kestin L and Vicini F: Long-term patterns of in-breast failure in patients with early stage breast cancer treated with breast-conserving therapy: A molecular based clonality evaluation. Am J Clin Oncol 33: 17-22, 2010.

22. Zhong Y,Lin Y, Shen S, Zhou Y, Mao F, Guan J and Sun Q: Expression of ALDH1 in breast invasive ductal carcinoma: An independent predictor of early tumor relapse. Cancer Cell Int 13: 60, 2013.

23. Ohi Y, Umekita Y, Yoshioka T, Souda M, Rai Y, Sagara Y, Sagara Y, Sagara Y and Tanimoto A: Aldehyde dehydrogenase 1 expression predicts poor prognosis in triple-negative breast cancer. Histopathology 59: 776-780, 2011.

24. Zhou L, Li K, Luo Y, Tian L, Wang M, Li C and Huang Q: Novel prognostic markers for patients with triple-negative breast cancer. Hum Pathol 44: 2180-2187, 2013.

25. Moja L, Tagliabue L, Balduzzi S, Parmelli E, Pistotti V, Guarneri $\mathrm{V}$ and D'Amico R: Trastuzumab containing regimens for early breast cancer. Cochrane Database Syst Rev 18: CD006243, 2012 . 